\title{
Improved Phenomenological Renormalization Schemes
}

\author{
M. A. Yurishchev \\ Vasilsursk Laboratory, Radiophysical Research Institute, 606263 \\ Vasilsursk, Nizhny Novgorod Region, Russia
}

\begin{abstract}
An analysis is made of various methods of phenomenological renormalization based on finite-size scaling equations for inverse correlation lengths, the singular part of the free energy density, and their derivatives. The analysis is made using two-dimensional Ising and Potts lattices and the three-dimensional Ising model. Variants of equations for the phenomenological renormalization group are obtained which ensure more rapid convergence than the conventionally used Nightingale phenomenological renormalization scheme. An estimate is obtained for the critical finite-size scaling amplitude of the internal energy in the threedimensional Ising model. It is shown that the two-dimensional Ising and Potts models contain no finite-size corrections to the internal energy so that the positions of the critical points for these models can be determined exactly from solutions for strips of finite width. It is also found that for the two-dimensional Ising model the scaling finite-size equation for the derivative of the inverse correlation length with respect to temperature gives the exact value of the thermal critical exponent.
\end{abstract}

PACS: 05.10.Cc, 64.60.Ak, 05.50.+q, 75.10.Hk 


\section{Introduction}

As we know [1], the renormalization group method lies at the basis of modern phase transition theory. In this approach, the critical points of a system are identified with the fixed points of renormalization group mappings. By linearizing the renormalization group transformations near a fixed point it is possible to find the critical exponents of the system of interest.

Many different specific realizations of the renormalization group procedure now exist. One of these realizations is the so-called phenomenological renormalization group proposed by Nightingale [2, 3] (see also the reviews [4, 5]). In this approach the scaling functional relationship between the correlation lengths of partially finite subsystems is interpreted as a renormalization group transformation. Combining this with expressions for the correlation lengths in terms of eigenvalues of the transfer matrices reduces the initial functional equation to a transcendental one and its solution gives an estimate for the critical temperature.

Dos Santos and Sneddon [6] noted that a phenomenological renormalization group can be constructed using not only the correlation length but any quantity having a power divergence at the phase transition point.

Binder [7, 8] suggested a variant of the phenomenological renormalization group based on using the moments of the distribution function of the order parameter; the moments can be expressed directly in terms of the susceptibility of the system. This method has been widely used in calculations using the Monte Carlo method. Recently, Itakura [9] expanded the Binder phenomenological renormalization scheme using linear combinations of different-order moments.

In the present study various phenomenological renormalization variants using a wide range of physical quantities (such as the free and internal energy, ordinary and nonlinear susceptibility, and so on) are used to calculate the critical coupling in the two-dimensional Ising and Potts models and also in the three-dimensional Ising model. An analysis can reveal the best renormalization strategies. Increasing the convergence is fundamentally important for threedimensional systems because as a result of the giant orders of the transfer matrices, the eigenvalue problem can only be solved for subsystems having extremely small transverse dimensions.

However, a study of the convergence of various types of renormalizations revealed equations which can be used to find exact values of the parameters of infinite systems (critical temperatures and in one case, critical exponent) using the characteristics of partially finite subsystems.

This article is constructed as follows. Section 2 contains equations for different variants of the phenomenological renormalization group. In Section 3 these equations are applied to the two-dimensional Ising and Potts models and in Section 4 they are applied to the three-dimensional Ising lattice. The conclusions reached are summarized briefly in the final section. 


\section{Equations for the phenomenological renormal- ization group}

In accordance with finite-size scaling theory 10, 11, 12, 13, the inverse correlation length $\kappa_{L}$ and the singular part of the reduced free energy density $f_{L}^{s}$ $\left(f_{L}^{s}=f_{L}-f_{\infty}\right.$, where $f_{L}$ and $f_{\infty}$ are the free energy densities of the subsystem and the total system, respectively) near the critical point $t=h=0$ $\left(t=K-K_{c}\right.$ is the "temperature" or more accurately the deviation from the critical coupling, and $h$ is the external field) satisfy the functional relationships

$$
\begin{gathered}
\kappa_{L}(t, h)=b^{-1} \kappa_{L / b}\left(t^{\prime}, h^{\prime}\right), \\
f_{L}^{s}(t, h)=b^{-d} f_{L / b}^{s}\left(t^{\prime}, h^{\prime}\right) .
\end{gathered}
$$

Here $d$ is the space dimension, $L$ is the characteristic linear size of the subsystem, $b=L / L^{\prime}$ is the coefficient of the scaling transformation. The renormalization group equations $t^{\prime}=t^{\prime}(t, h)$ and $h^{\prime}=h^{\prime}(t, h)$ linearized near the fixed point $t=h=0$ have the form

$$
\begin{aligned}
t^{\prime} & =b^{y_{t}} t+O\left(t^{2}\right), \\
h^{\prime} & =b^{y_{h}} h+O\left(h^{2}\right),
\end{aligned}
$$

where $y_{t}$ and $y_{h}$ are the thermal and magnetic critical exponents of the system.

In the Nightingale approach [2, 3, , 4, 5] the relationship (11) is treated as a renormalization group mapping $(t, h) \rightarrow\left(t^{\prime}, h^{\prime}\right)$. In this case, the inverse correlation length of the spin-spin correlation function is given by the standard expression

$$
\kappa_{L}=\ln \left(\lambda_{1}^{(L)} /\left|\lambda_{2}^{(L)}\right|\right),
$$

where $\lambda_{1}^{(L)}$ and $\lambda_{2}^{(L)}$ are the largest and second largest eigenvalues of the transfer matrix of the subsystem, respectively. As a result, for systems with phase diagram symmetry $h \rightarrow-h$ (and thus $h_{c}=0$; we only consider these systems here) the only unknown coordinate of the critical point can be determined from the transcendental equation

$$
L \kappa_{L}\left(K_{c}\right)=(L-1) \kappa_{L-1}\left(K_{c}\right)
$$

(in order to maximize the accuracy of the estimate $K_{c}$ it is usually assumed that $\left.L^{\prime}=L-1\right)$. Nightingale described his renormalization method as "phenomenological" 33] (see also 14) since it uses a scaling finite-size equation which is not derived microscopically using the method.

Quite clearly, the phenomenological renormalization $(t, h) \rightarrow\left(t^{\prime}, h^{\prime}\right)$ can be made with equal justification using relation (2). In this case, we obtain the following equation to determine the critical coupling $K_{c}$

$$
L^{d} f_{L}^{s}\left(K_{c}\right)=(L-1)^{d} f_{L-1}^{s}\left(K_{c}\right) .
$$


The free energy density of the partially finite subsystem $L^{d-1} \times \infty$ is then calculated using the formula

$$
f_{L}=L^{1-d} \ln \lambda_{1}^{(L)},
$$

and the "background" $f_{\infty}$ is taken from outside.

Differentiating expressions (1) and (2) $m$ times with respect to temperature and $n$ times with respect to the field allowing for (3) and (4), we obtain [15]

$$
\begin{aligned}
L^{1-m y_{t}-n y_{h}} \kappa_{L}^{(m, n)}(t, h) & =\left(L^{\prime}\right)^{1-m y_{t}-n y_{h}} \kappa_{L^{\prime}}^{(m, n)}\left(t^{\prime}, h^{\prime}\right), \\
L^{d-m y_{t}-n y_{h}} f_{L}^{s(m, n)}(t, h) & =\left(L^{\prime}\right)^{d-m y_{t}-n y_{h}} f_{L^{\prime}}^{s(m, n)}\left(t^{\prime}, h^{\prime}\right) .
\end{aligned}
$$

These relationships or some combinations thereof can also be considered as implicit renormalization group transformations. For large subsystem sizes all equations of this type should give the same results. However, for small L which we need to deal with in practice, the various renormalization group equations yield estimates of different accuracy. As we shall see, this circumstance means that it is possible to construct phenomenological renormalization schemes which give faster convergence than that achieved in conventionally used realizations of the phenomenological renormalization group method.

In addition to equations (6) and (7), in the present study we also give estimates of the critical coupling obtained from the following renormalization group equations [taking those ratios (9) and (10), from which the unknown exponents $y_{t}$ and $y_{h}$ are dropped a priori]:

$$
\left.\frac{\chi_{L}^{(4)}}{L^{d} \chi_{L}^{2}}\right|_{K_{c}}=\left.\frac{\chi_{L-1}^{(4)}}{(L-1)^{d} \chi_{L-1}^{2}}\right|_{K_{c}},
$$

where $\chi_{L}=\partial^{2} f_{L} /\left.\partial h^{2}\right|_{h=0}=f_{L}^{s(0,2)}$ is the susceptibility and $\chi_{L}^{(4)}=\partial^{4} f_{L} /\left.\partial h^{4}\right|_{h=0}=$ $f_{L}^{s(0,4)}$ is the nonlinear susceptibility (equation (11) corresponds to the Binder phenomenological renormalization [7, 8 ]);

$$
\begin{aligned}
L^{2-d}\left(\kappa_{L}^{(1)}\right)^{2} / \chi_{L} & =(L-1)^{2-d}\left(\kappa_{L-1}^{(1)}\right)^{2} / \chi_{L-1} ; \\
L^{1-d} \kappa_{L}^{(2)} / \chi_{L} & =(L-1)^{1-d} \kappa_{L-1}^{(2)} / \chi_{L-1} ; \\
L^{1-2 d} \kappa_{L}^{(4)} / \chi_{L}^{2} & =(L-1)^{1-2 d} \kappa_{L-1}^{(4)} / \chi_{L-1}^{2} .
\end{aligned}
$$

Here we have $\kappa_{L}^{(n)}=\partial^{n} \kappa_{L} /\left.\partial h^{n}\right|_{h=0}=\kappa_{L}^{(0, n)}(K, 0)$. Formulas for the derivatives of the inverse correlation length and the free energy with respect to $h$, expressed in terms of the eigenvalues and eigenvectors of the transfer matrices, suitable for programming are given in [16, 17.

For better clarity, equations (6), (7), (11), (12), (13), and (14) will be denoted by the mnemonic symbols " $\kappa$ ", " $f s ", " \chi \chi^{(4)} / \chi^{2 ",}, "\left(\kappa^{(1)}\right)^{2} / \chi, " \kappa^{(2)} / \chi$ ", and " $\kappa^{(4)} / \chi^{2}$ ", respectively when the numerical data are given in the tables. 


\section{Two-dimensional systems}

We shall first test various types of phenomenological renormalization group equations on the two-dimensional Ising and Potts models, for which the positions of the critical points are known in exact analytical form 18,19 .

\subsection{Ising model}

We shall start with the most studied system, the Ising model. Table 1 gives results of our calculations of the critical coupling for a simple square Ising lattice. The calculations were made for $L \times \infty$ strips having a periodic boundary condition in the transverse direction. Estimates are given for $(L-1, L)$ pairs with $L \leq 5$. For a $(3,4)$ pair the relative errors of the estimates are also given in parentheses; their sign indicates whether they are lower (-) or upper $(+)$ estimates. The types of renormalization group equations used are indicated in the first column. We recall that in this model we have

$$
K_{c}=\frac{1}{2} \ln (1+\sqrt{2}), \quad f_{\infty}=\frac{2 G}{\pi}+\frac{1}{2} \ln 2
$$

( $G$ is the Catalan constant) [20].

It can be seen from the data presented in Table 1 that the best lower estimate of $K_{c}$ is obtained from equation (14). The Binder scheme gives slightly inferior results. This approach, which is usually used for Monte Carlo simulations, as we noted in the Introduction [because it does not have formulas of the type (阿) for the inverse correlation length], was used in the transfer matrix variant in [21]. The Nightingale renormalization (first row in Table 1) which is almost universal (except perhaps for the study just mentioned [21]) is used for calculations using the transfer matrix method and is only third in terms of accuracy among the lower estimates.

It is worth noting that we also succeeded in obtaining phenomenological renormalization group equations which also yield upper estimates of $K_{c}$ (the two lower rows in Table 1). Of these two estimates the renormalization scheme using the singular part of the free energy density [equation(7)] gives betterquality results. The values obtained using this scheme are at the same time the best in terms of absolute value among all the lower and upper estimates.

Unfortunately the renormalization procedure using the singular part of the free energy requires an advance knowledge of the critical free energy of the system $f_{\infty}$. In order to overcome this constraint, instead of the two strips required to construct the renormalization group equation, we take three. In other words we shall take the triads $\left(L_{1}, L_{2}, L_{3}\right)$; an additional relationship can eliminate the parameter $f_{\infty}$. For the free energy densities of the three subsystems at the critical point we have

$$
f_{L_{1}}=f_{\infty}+A_{f} / L_{1}^{d}, \quad f_{L_{2}}=f_{\infty}+A_{f} / L_{2}^{d}, \quad f_{L_{3}}=f_{\infty}+A_{f} / L_{3}^{d} .
$$


Here $A_{f}=L^{d} f_{L}^{s}(0,0)$ is the critical finite-size scaling amplitude of the singular part of the free energy density. Eliminating the parameters $f_{\infty}$ and $A_{f}$ from the system (15), we arrive at the equation

$$
\mathcal{F}(K)=F(K)-\frac{L_{2}^{d}-L_{1}^{d}}{L_{3}^{d}-L_{2}^{d}}\left(\frac{L_{3}}{L_{1}}\right)^{d}=0
$$

where

$$
F(K)=\frac{f_{L_{1}}-f_{L_{2}}}{f_{L_{2}}-f_{L_{3}}} .
$$

Equation (16) can apparently be used to determine the position of the critical point but it has no real roots.

All the eigenvalues of the transfer matrix of an $L \times \infty$ Ising cylinder are known in exact analytic form for any $L$ [20, 22]. The largest eigenvalue is

$$
\lambda_{1}^{(L)}=(2 \sinh 2 K)^{L / 2} \exp \left[\left(\gamma_{1}+\gamma_{3}+\ldots+\gamma_{2 L-1}\right) / 2\right],
$$

where $\gamma_{r}$ are the positive solutions of the equations

$$
\cosh \gamma_{r}=\cosh 2 K \operatorname{coth} 2 K-\cos (\pi r / L) .
$$

Using formula (8) we then obtain expressions for the free energy densities $f_{L_{i}}$ $(i=1,2,3)$ contained in (17).

Subsequent calculations using these formulas show that the curves $\mathcal{F}(K)$ do not intersect the abscissa and thus the equation $\mathcal{F}(K)=0$ has no real solutions (see Fig. 1). However, it is noticeable that for various choices of parameters $L_{1}, L_{2}$, and $L_{3}$ the function $\mathcal{F}(K)$ [and therefore $F(K)$ ] has an extremum positioned at the exact value of the critical coupling. Differentiating relation (17) with respect to $K$, we arrive at the equation

$$
\left(u_{L_{1}}-u_{L_{2}}\right)\left(f_{L_{2}}-f_{L_{3}}\right)=\left(u_{L_{2}}-u_{L_{3}}\right)\left(f_{L_{1}}-f_{L_{2}}\right),
$$

where $u_{L}=\partial f_{L} / \partial K$ is the internal energy of the subsystem. By using this equation or finding the extremum of the function $F(K)$ we can extract exact values of the critical temperatures for infinite systems (at any rate for some) from the characteristics of their finite subsystems. (This is the desired aim of any finite-size theory.)

Equation (20) can be approached from several positions. We shall analyze expression (10) in the first derivative with respect to $K(m=1, n=0)$. For a fixed point this renormalization group transformation gives

$$
L^{d-y_{t}} u_{L}^{s}\left(K_{c}\right)=\left(L^{\prime}\right)^{d-y_{t}} u_{L^{\prime}}^{s}\left(K_{c}\right),
$$

where $u_{L}^{s}=u_{L}-u_{\infty}$ is the singular part of the internal energy density. It follows from (19) that

$$
\partial \gamma_{r} /\left.\partial K\right|_{K=K_{c}}=0 \quad(r \neq 0)
$$


(in this context see Fig. 118 in the book 234). After differentiating we find that $u_{L}\left(K_{c}\right)=\sqrt{2}$ regardless of $L$. In other words, all the finite-size corrections to the background $u_{\infty}(=\sqrt{2}[20])$ are zero, i.e., the singular part of the internal energy is completely absent. Consequently, we obtain

$$
u_{L}^{s}\left(K_{c}\right)=0 .
$$

This property of the model leads to equations (20) and (21) being satisfied.

We shall now analyze the first derivative with respect to temperature of the other fundamental equation in finite-size scaling theory, i.e., equation (1).

The second eigenvalue of the transfer matrix of the Ising model in terms of value is given by

$$
\lambda_{2}^{(L)}=(2 \sinh 2 K)^{L / 2} \exp \left[\left(\gamma_{0}+\gamma_{2}+\ldots+\gamma_{2 L-2}\right) / 2\right] .
$$

Equations (5), (18), (19), and (24) can be used to find an analytic expression for the inverse correlation length. Taking the first derivative with respect to $K$ we obtain the dependencies $\dot{\kappa}_{L}(K) \equiv \partial \kappa_{L} / \partial K$ for various $L$. All these dependencies have a common point of self-intersection and this is positioned at the exact value of the critical coupling: $\dot{\kappa}_{L}\left(K_{c}\right)=-2$ for all $L=1,2, \ldots$ (i.e. in an infinite lattice $\dot{\kappa}_{\infty}=-2$ ). Thus we have

$$
\dot{\kappa}_{L}\left(K_{c}\right)=\dot{\kappa}_{L^{\prime}}\left(K_{c}\right) .
$$

Reversing this reasoning, we conclude that equation (25) can also be used to determine the exact position of the critical point of an infinite two-dimensional Ising lattice using the solutions for strips of finite width.

Moreover, in accordance with relation (9) for $m=1$ and $n=0$ we have

$$
y_{t}=1+\frac{\ln \left(\dot{\kappa}_{L} / \dot{\kappa}_{L^{\prime}}\right)}{\ln \left(L / L^{\prime}\right)}
$$

Consequently, this finite-size expression taking into account condition (25) gives an exact value for the thermal critical exponent of the two-dimensional Ising model: $y_{t}=1$.

For the Ising model equation (25) can be generalized to the form

$$
\left(\dot{\kappa}_{L_{1}}-\dot{\kappa}_{L_{2}}\right)\left(\kappa_{L_{2}}-\kappa_{L_{3}}\right)=\left(\dot{\kappa}_{L_{2}}-\dot{\kappa}_{L_{3}}\right)\left(\kappa_{L_{1}}-\kappa_{L_{2}}\right) .
$$

and the problem of determining $K_{c}$ can be reduced to searching for the extremum of the function

$$
\Phi=\frac{\kappa_{L_{1}}-\kappa_{L_{2}}}{\kappa_{L_{2}}-\kappa_{L_{3}}}
$$




\subsection{Potts model}

In the two-dimensional q-state Potts model for an isotropic square-cell lattice the critical coupling is $K_{c}=\ln (1+\sqrt{q})$ [24]. Table 2 contains estimates of $K_{c}$ obtained by us using various constructions of the phenomenological renormalization group for the Potts model with three states $(q=3)$. The free energy background in this model is given by [25, 26]

$$
f_{\infty}=4 G / 3 \pi+\ln (2 \sqrt{3})+\frac{1}{3} \ln (2+\sqrt{3}) .
$$

An analysis of the data given in Table 2 shows that equations (7) and (12) give substantially better-quality estimates than the Nightingale approach (first row in the table). The renormalization group equation based on the scaling relationship for the singular part of the free energy again gives the smallest errors in terms of absolute value.

Calculations made for $L \times \infty$ strips with a periodic boundary condition in the transverse direction then uniquely indicate that in the two-dimensional q-state Potts model all finite-size corrections to the internal energy $\left(u_{\infty}=1+1 / \sqrt{q}\right.$ [18) are also absent at the critical point. Consequently, the equation

$$
u_{L}\left(K_{c}\right)=u_{L^{\prime}}\left(K_{c}\right)
$$

gives the exact value of $K_{c}$. Satisfying condition (30) then has the result that equation (20) is valid and thus the extremum of the function (17) is positioned strictly at the exact value of the critical coupling. It should be noted that in computer calculations it is more logical to search directly for the extremum of the function than to differentiate the free energy numerically and then solve the transcendental equation (30) numerically.

Equation (30) for the pair $(1,2)$ was first obtained by Wosiek from his postulated maximum principle for the normalized moments of transfer matrices [27] (see also [28]). In [29] the Wosiek equation was then generalized to the form (30). Our equation (20) is a further generalization of (30).

Note that in the Potts model the curves $\dot{\kappa}_{L}(K)$ also intersect. However, unlike the Ising model the points of intersection are not now positioned at the exact value of $K_{c}$. At the exact value of the critical coupling the functions $\dot{\kappa}_{L}(K)$ have different values for various $L$. Unfortunately the scaling formula (26) then only gives approximate estimates of the thermal critical exponent which, for example for the three-site Potts model is $y_{t}=6 / 5$.

Returning to the two-dimensional Ising model we can confirm that a complete system of equations of the type (30) is in fact obtained for this model:

$$
u_{L}^{j}\left(K_{c}\right)=u_{L^{\prime}}^{j}\left(K_{c}\right),
$$

where $u_{L}^{j}=\partial f_{L}^{j} / \partial K$ are internal energy levels; this term was taken by analogy

with the "free energy levels" $f_{L}^{j}=L^{1-d} \ln \lambda_{j}^{(L)}, j=1,2, \ldots$ (see, for example $[30])$. 


\section{Three-dimensional Ising lattice}

We shall now consider a three-dimensional Ising model for a simple cubic lattice. Unlike the two-dimensional Ising model, which is solved analytically over the entire temperature range, or the two-dimensional Potts model which is solved exactly at a single point, the critical point, for the three-dimensional Ising model we are forced to confine ourselves to numerical estimates. The highest-quality estimates for the critical coupling and the free energy background have now been obtained by Monte Carlo simulation for $L \times L \times L$ cubes: $K_{c}=0.22165459(10)$ [31] and $f_{\infty}=0.77790(2)$ [32].

Table 3 gives our results for the three-dimensional Ising model. The renormalizations were made for $L \times L \times \infty$ parallelepipeds with periodic boundary conditions in both transverse directions. The calculations were made for subsystems with side lengths $L \leq 4$, i.e. for transfer matrices having dimensions up to $65536 \times 65536$.

We can see from Table 3 that as in the two-dimensional case, the best lower estimates of $K_{c}$ are provided by the renormalization group equation (14). On comparing the lower and upper estimates we can conclude that the best of all the results is again given by renormalizing the singular part of the free energy.

In the three-dimensional case the amplitudes of the finite-size corrections to the internal energy at the critical point are no longer zero. In accordance with the scaling equation (21) we have

$$
u_{L}\left(K_{c}\right)=u_{\infty}+A_{u} L^{-d+y_{t}}+\ldots,
$$

where $A_{u}$ is the amplitude of the leading finite-size term in the internal energy density of the system at the critical point. Calculations using formula (32) for a $4 \times 4 \times \infty$ cluster using the values $u_{\infty}=0.990637(47)$ 33, 34] and $y_{t}=1.5865(14)$ 31 give $A_{u} \simeq 1.19$.

Since $A_{u} \neq 0$, equation (30) now only reproduces an approximate value of $K_{c}$. Also equation (20) is not satisfied exactly. Calculations of the position of the minimum of the function (17) for the triad $(2,3,4)$ yield the estimate $K_{c}=$ 0.233003 . Unfortunately, the accuracy of this estimate $(5.1 \%)$ is significantly inferior to the errors of the estimates obtained from the other renormalization schemes (see Table 3).

\section{Conclusions}

Nontrivial models with known values of the critical coupling have been used to study phenomenological renormalization schemes which for the same subsystem sizes give more accurate results than those used so far. We established that for Ising models the renormalization group equations using combinations $L^{1-2 d} \kappa_{L}^{(4)} / \chi_{L}^{2}$ and $L^{1-d} \kappa_{L}^{(2)} / \chi_{L}$, give uniformly converging bilateral estimates 
of $K_{c}$ where the errors (in the three-dimensional, most important case) are less than half those obtained in the Nightingale method.

Another result of this study is related to the exact results. We have shown that by making the amplitudes of the finite-size corrections to the internal energy vanish, exact values of the critical temperatures can be determined for the infinite two-dimensional Ising and Potts models from the solutions for their partially finite subsystems. In addition, we have observed that for the twodimensional Ising model the temperature derivative of the inverse correlation length has absolutely no finite-size corrections. This means that it is also possible to find the exact value of the thermal critical exponent for this model from the finite-size equation.

Both the conclusions reached from these results have practical applications but separate analysis is required to develop them.

\section{Acknowledgments}

The author thanks A. A. Belavin for useful discussions and valuable comments. Thanks are also due to J. Wosiek for showing interest in this work. This work was supported financially by the Russian Foundation for Basic Research (project no. 99-02-16472). 


\section{References}

[1] S. Ma, Modern Theory of Critical Phenomena, (Benjamin, Reading, Mass., 1976; Mir, Moscow, 1980).

[2] M. P. Nightingale, Physica A 83, 561 (1976).

[3] M. P. Nightingale, Proc. Kon. Ned. Akad. Wet. B 82, 235 (1979).

[4] P. Nightingale, J. Appl. Phys. 53, 7927 (1982).

[5] M. P. Nightingale, in Finite Size Scaling and Numerical Simulation of Statistical Systems, ed. by V. Privman, World Scientific, Singapore (1990), p. 287.

[6] R. R. dos Santos and L. Sneddon, Phys. Rev. B 23, 3541 (1981).

[7] K. Binder, Phys. Rev. Lett. 47, 693 (1981).

[8] K. Binder, Z. Phys. B 43, 119 (1981).

[9] M. Itakura, Preprint cond-mat/9611174.

[10] M. Fisher, in International School of "Enrico Fermi" on Critical Phenomena, 1970, Ed. by M. S. Green (Academic, New York, 1971); F. J. Dyson, E. W. Montroll, M. Kac, and M. Fisher, Ustojchivost' $i$ Fazovye Perekhody (Mir, Moscow, 1973), s. 245.

[11] M. E. Fisher and M. N. Barber, Phys. Rev. Lett. 28, 1516 (1972).

[12] M. N. Barber, in Phase Transitions and Critical Phenomena, ed. by C. Domb and J. L. Lebowitz, Academic Press, London (1983), Vol. 8, p. 145 .

[13] V.Privman, in Finite Size Scaling and Numerical Simulation of Statistical Systems, ed. by V. Privman, World Scientific, Singapore (1990), p. 1.

[14] W. Kinzel and M. Schick, Phys. Rev. B 23, 3435 (1981).

[15] M. A. Yurishchev, Nucl. Phys. B (Proc. Suppl.) 83-84, 727 (2000).

[16] M. A. Yurishchev, Phys. Rev. B 50, 13533 (1994).

[17] M. A. Yurishchev, Phys. Rev. E 55, 3915 (1997).

[18] R. J. Baxter, Exactly Solved Models in Statistical Mechanics (Academic, New York, 1982; Mir, Moscow, 1985).

[19] F. Y. Wu, Rev. Mod. Phys. 54, 235 (1982).

[20] L. Onsager, Phys. Rev. 65, 117 (1944). 
[21] H. Saleur and B. Derrida, J. Phys. (Paris) 46, 1043 (1985).

[22] B. Kaufman, Phys. Rev. 76, 1232 (1949).

[23] K. Huang, Statistical Mechanics (Wiley, New York, 1963; Mir, Moscow, 1966).

[24] R. B. Potts, Proc. Cambr. Phil. Soc. 48, 106 (1952).

[25] R. J. Baxter, J. Phys. A 6, L445 (1973).

[26] R. J. Baxter, Proc. Roy. Soc. London A 383, 43 (1982).

[27] J. Wosiek, Phys. Rev. B 49, 15023 (1994).

[28] Z. Burda and J. Wosiek, Nucl. Phys. B (Proc. Suppl.) 34, 677 (1994).

[29] A. Pelizzola, Phys. Rev. B 51, 12005 (1995).

[30] V. Privman and M. E. Fisher, Phys. Rev. B 30, 322 (1984).

[31] H. W. J. Blöte, L. N. Shchur, and A. L. Talapov, Int. J. Mod. Phys. C 10, 1137 (1999).

[32] K. K. Mon, Phys. Rev. B 39, 467 (1989).

[33] M. Hasenbusch and K. Pinn, Nucl. Phys. B (Proc. Suppl.) 63, 619 (1998).

[34] M. Hasenbusch and K. Pinn, J. Phys. A 31, 6157 (1998). 
Table 1: Estimates of $K_{c}$ for a two-dimensional square Ising lattice; $K_{c}^{\text {exact }}=$ $\underline{0.440686 \ldots}$

\begin{tabular}{clll}
\hline eq. & $(2,3)$ & $(3,4)$ & $(4,5)$ \\
\hline$(\kappa)$ & 0.42236 & $0.43088(-2.23 \%)$ & 0.43595 \\
$\left(\chi^{(4)} / \chi^{2}\right)$ & 0.42593 & $0.43242(-1.88 \%)$ & 0.43672 \\
$\left(\kappa^{(4)} / \chi^{2}\right)$ & 0.42596 & $0.43243(-1.87 \%)$ & 0.43673 \\
$\left(f^{s}\right)$ & 0.44324 & $0.44168(+0.23 \%)$ & 0.44105 \\
$\left(\kappa^{(2)} / \chi\right)$ & 0.47420 & $0.45153(+2.64 \%)$ & 0.44626 \\
\hline
\end{tabular}

Table 2: Estimates of $K_{c}$ for a two-dimensional square three-site Potts lattice; $\underline{K_{c}^{\text {exact }}=1.005052 \ldots}$

\begin{tabular}{clll}
\hline eq. & $(2,3)$ & $(3,4)$ & $(4,5)$ \\
\hline$(\kappa)$ & 0.96248 & $0.98350(-2.1 \%)$ & 0.99467 \\
$\left(\kappa^{(1)^{2}} / \chi\right)$ & 0.99311 & $0.99920(-0.6 \%)$ & 1.00380 \\
$\left(f^{s}\right)$ & 1.00927 & $1.00667(+0.2 \%)$ & 1.00565 \\
\hline
\end{tabular}

Table 3: Estimates of $K_{c}$ for a three-dimensional simple cubic Ising lattice; $K_{c}^{\text {exact }}=0.22165459(10)$

\begin{tabular}{cll}
\hline eq. & $(2,3)$ & $(3,4)$ \\
\hline$(\kappa)$ & 0.21340 & $0.21826(-1.53 \%)$ \\
$\left(\chi^{(4)} / \chi^{2}\right)$ & 0.21823 & $0.22002(-0.74 \%)$ \\
$\left(\kappa^{(4)} / \chi^{2}\right)$ & 0.21824 & $0.22006(-0.72 \%)$ \\
$\left(f^{s}\right)$ & 0.22354 & $0.22236(+0.32 \%)$ \\
$\left(\kappa^{(2)} / \chi\right)$ & 0.22658 & $0.22323(+0.71 \%)$ \\
\hline
\end{tabular}




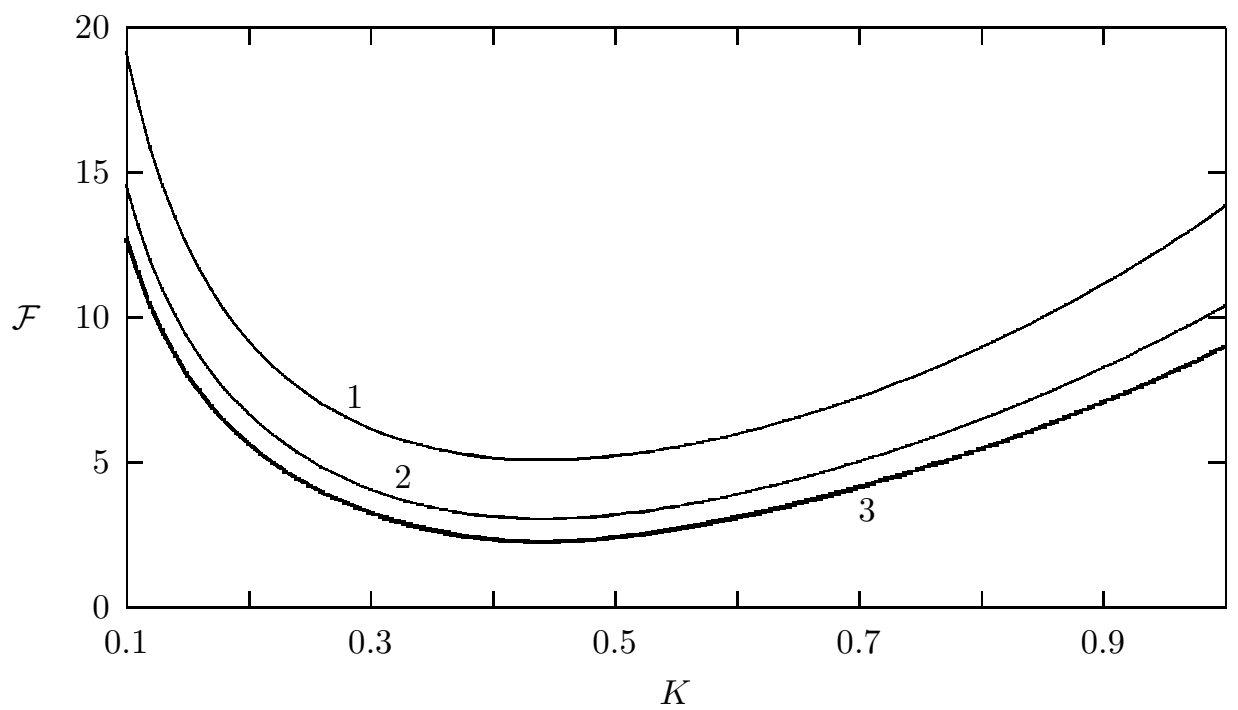

Figure 1: Behavior of the function $\mathcal{F}(K)$ for triads of Ising strip clusters $(1,2,3),(2,3,4)$, and $(3,4,5)$ given by curves 1,2 , and 3 , respectively. In all cases the minimum of the function is positioned at the exact value $K_{c}=$ $\frac{1}{2} \ln (1+\sqrt{2})=0.440686 \ldots$. 